\title{
COVID-19 and
}

\section{Community Dental}

Services: The

\section{challenges ahead}

David Westgarth

Editor, BDJ In Practice

\section{Introduction}

With more than a gaze being cast towards 'what will dentistry look like once we can start to treat patients', at the time of writing much of the focus appears to be on high street dentistry. These are - in the main patients that can visit a dental practice with the minimum of fuss.

But what about those who cannot? Patients treated within the community dental services do not have the luxuries afforded to those treated in a 'regular' dental practice. Paediatric patients with rampant caries have their teeth removed under general anaesthetic in hospital. Domiciliary patients have very specific needs. Special care patients often require more time during their appointment and a flexible approach. The same applies to frail older people.

And yet the fundamental approaches to treating these cohorts are likely to look very different once dentistry resumes. Could the challenging nature of treating patients within community dental services be even more difficult in a post-COVID-19 world?

\section{A new-look service?}

Changes are inevitable. You only need to look at the discussions around social distancing, PPE, AGPs and standard operating procedures to know that. Jenny Harris, Consultant in Community Paediatric 


\section{Key points}

$\rightarrow$ Dental services will have to adapt

$\rightarrow$ Could the CDS face the biggest changes post-COVID-19?

$\rightarrow$ To what extent will patients suffer from these changes?

Dentistry, Community \& Special Care Dentistry, Sheffield Teaching Hospitals NHS Foundation Trust and BSPD Vice President Designate, believes a return to normal may not be the most suitable approach.

'Services are going to look very different over the next few months as we adapt to the changing situation', she said. 'In our specialty we're talking about 'Building Back Better' rather than a return to normal. We have an opportunity to take stock and prioritise what really matters. Colleagues around the country have been contributing ideas to national guidance on the pandemic 'recovery' phase: guidance that focuses on what should take priority. Children experiencing dental pain should be the first in the queue so, amongst other things, that means we will need to restore access to general anaesthesia at an early stage across the whole country. We are also thinking about the needs of medically complex children.

'Yet we must not lose sight of the importance of preventing dental caries in the first place. What a difference we could make if we radically upscaled dental prevention! The pandemic has stimulated a spirit of collective enthusiasm and sense of immediacy for sharing ideas.'

Linzi Maybin, founder of Happy Teeth Outreach, identified some of the challenges facing the profession. She said: 'Over the last 10 weeks COVID-19 has radically changed the future of dentistry. From social distancing measures in dental waiting rooms to dentists wearing more robust PPE when providing dental treatment. Personally, I have seen all Happy Teeth outreach projects cancelled. But even though we cannot provide a 'normal' outreach programme, we as an organisation have worked hard to maintain strong relationships with the schools and refugee centres during this pandemic to continue to promote good oral health for children.

With COVID-19 sweeping through care homes, I asked Mili Doshi, Consultant in Special Care Dentistry and Clinical Lead 
for Mouth Care Matters and Vicki Jones, Clinical Director of CDS for Aneurin Bevan University Health Board and Chair of British Society of Gerodontology, about the challenges they foresee in providing dental care for older people.

Vicki said: 'COVID-19 has hit the older vulnerable population the hardest and especially devastating for many people living in care homes. It brings into focus the concerns we have previously expressed about the oral health of older people. In 2019 the CQC report 'Smiling Matters' found alarmingly poor standards of mouth care for care home residents and limited access to dentistry especially for urgent dental conditions in England.

'Levels for NHS commissioning for domiciliary dental care have reduced over the years which will impact on access to oral health care for the most vulnerable older population. A freedom of information request by the BDA last year found domiciliary services in England are equivalent to providing coverage to under $1.3 \%$ of the population whose activity is significantly limited by disability or ill health. Currently, Community Dental Services across the UK are been providing urgent dental care for shielding and high risk groups including older people, this is
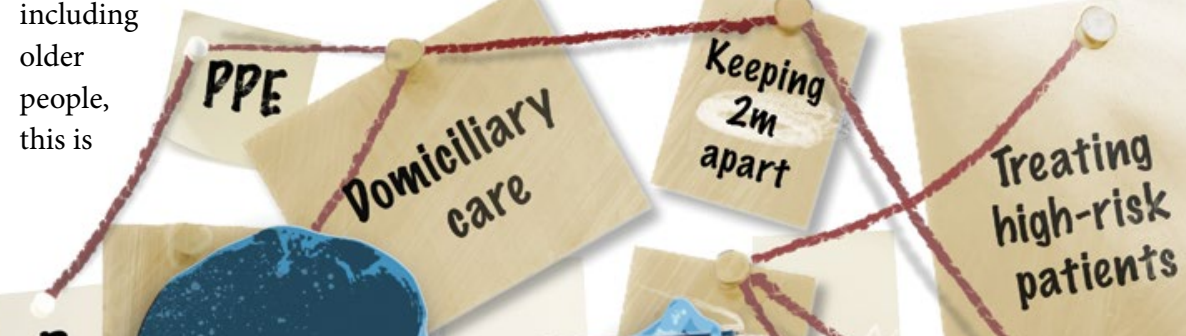

likely to continue through the de-escalation phases of COVID-19 pandemic but, as dental services start to resume, questions have to be asked whether this is a sustainable model in the long term.'

Mili added: 'We know the older population are increasingly retaining their own teeth with complex dental restorative care, and as a result have very specific challenges. Older people tend to develop dental disease more rapidly than the general population as a result of dry mouth, high sugar diets and lack of regular mouth care, and this can lead to severe enduring pain and infection. Poor oral health can impact on their ability to eat and drink leading to malnutrition, difficulty communicating and a poorer quality of life especially for those who have cognitive impairment and find it difficult to vocalise dental pain or discomfort.

'When the time comes for dental services to resume through the COVID-19 deescalation phases, there will continue to be a requirement to maintain specific clinics or appointments for shielding and highrisk groups of patients while meeting these needs. This will put an added pressure on community dental services who will have an existing backlog of special care children and adults that also require dental treatment. There is, and will be, an opportunity for better collaboration between community dental services, general dental services and other primary care teams for integrated oral care for older people. As Jenny mentioned, it is about building a better model, not simply returning to what was the normal.'

\section{Practitioners, patients and procedures} As a child I was always terrified of the scene in ET when the special unit dressed in full hazmat suits burst into Elliott's house looking for the creature. And that was TV, and fictional.

What relevance does this have to anything, you may ask. Well, having seen images of NHS workers in full PPE with huge facemasks and shields, it conjures memories of how I felt; how scared I was. And you can imagine a scenario in an anxious child where things begin to snowball; their anxiety, lack of cooperation and limited access to services could result in more dental infections. In turn this would result in delayed treatment and could lead to abscesses or facial swellings. Consequently, this could have a knock-on effect with the acute maxillofacial setting if more children have a delay in routine dental treatment. This means increased A\&E attendances for tooth decay, particularly if local measures of antibiotics prescriptions are not successful in reducing the swelling.

This in itself presents a question: where will the main challenges lie - with practitioners, procedures or patients?

'One of paediatric dentistry's first concerns was that children might be frightened by dentists dressed in full PPE', Jenny explained. 'So, very early in the pandemic, colleagues designed new illustrated leaflets to help prepare families in advance and preempt these anxieties. BSPD has assembled a raft of these resources to download from our website. ${ }^{1}$

'Children are both remarkably resilient and not as likely as adults to become severely ill with COVID-19 so the direct effects of the pandemic on them may not be so obvious. However, they will be suffering indirectly as a result of family stress, job loss or bereavement, and the impact will be greatest for the estimated 4 million UK children who live in poverty.

'We shouldn't underestimate what a challenge lockdown may have been for the workforce. So much of our job satisfaction comes from relationships with our patients 
and their parents; that ongoing connection

with each other, as we face the ups and downs of life, which was snatched away when face-to-face dental care stopped. I've never known a time in my life when I've had so little contact with children. And yet it's bound to feel very odd for a time once we go back. I think it's likely that some of us will be very keen to get back to normal as soon as possible, yet others will be extremely nervous about that. We will need to pull together and support each other.

'As for dental procedures, this gives us an opportunity to carefully choose operative techniques that provide predictably good outcomes, ideally with fewer visits and with the minimum of aerosol-generating exposures. Obvious ones that come to mind are Hall Technique pre-formed metal crowns as an effective and child-friendly way of treating primary tooth caries and single-visit obturation techniques for non-vital immature teeth. At my clinic we had already ordered some silver diamine fluoride (SDF) to start using to arrest caries, particularly in primary incisors, to reduce the need for extractions and general anaesthesia but it didn't arrive until the week we stopped seeing patients. I'm planning to open that on the first day back!'

Besides the obvious concerns about the availability of PPE - for both patient and practitioner - Linzi echoed Jenny's sentiment that there are multiple areas within the community that will be challenged depending on the length of pandemic's impact on dentistry.

'It is unusual for dental practices and staff in the short term to get their head around limiting contact we have with patients', she said. 'However, this is the new norm. During the 'recovery' stage of this pandemic there may initially be fewer patients seen on a daily basis but this is ensuring safety for all.

'It is all well and good suggesting dentistry reopens, but you only need to look at how challenging it can be for our very young patients in pain with dental decay. These children may be too un-cooperative or anxious to accept dental treatment awake. In this situation, a general anaesthetic is the treatment of choice, yet many of the UK's routine paediatric general anaesthetic procedures have been postponed due to the pandemic. Initially I was concerned children experiencing dental pain would not be treated, but I have seen research on urgent and emergency dental care centres that suggest they have effectively managed problems.'

As far as Mili and Vicki are concerned, their major worry remains the health of their patients.

'Due to fear of contracting COVID-19, these vulnerable and shielding older people, are not accessing dental care when they need to which could be detrimental or even fatal to their general health', Vicki suggested. 'There has been a marked decrease of patients being referred for urgent suspected cancer assessments for example. Local health communications teams can reassure the older public that should they require urgent dental care that the infection control and physical distancing will be in place so they are at reduced risk for contracting COVID- 19 . Much of the communication going out to the public is on social media and the internet, older people may not have access to a smart phone or computer/iPad.

\section{'There is, and will be, an opportunity for better collaboration between community dental services, general dental services and other primary care teams for integrated oral care for older people.'}

'You also need to consider that phone triaging can be difficult for people with cognitive conditions such as dementia who are unable to verbally articulate if they have oral pain. We often rely on carers noticing changes in eating or behavioural patterns, facial swellings and fractured/sharp teeth. Families are usually the first to raise dental concerns and organise dental appointments. The pandemic has resulted in most care homes not allowing any visitors, to reduce the risk of virus transmission. This is the same for relatives not being able to visit those living in their own home for isolation reasons and therefore that support in contacting dental services is reduced.'

According to Mili, if someone in a care home requires urgent dental care, there are additional areas beyond these to consider.

'Many care homes are avoiding residents leaving the home and people entering to minimise the risk of contracting the virus. The use of AAA may be useful initially for a patient but this has to be monitored by the dental team should a patient have serious underlying health conditions. People living alone or with other people who are at high risk may rely on friends or family to take them to dental appointments, which may not be possible. For some patients where the dental team has established that the dental treatment is relatively straightforward, for example extraction of a mobile tooth, it may be more appropriate to provide domiciliary care. Each health board/trust area should have local SOPs for domiciliary dental care that includes appropriate PPE be used to minimise the risk of the dental team being asymptomatic carriers introducing infection to an isolating care home.

'Regardless of the dental setting, when wearing a mask and full-face visor does make it harder to communicate and express emotion. Many older people will also have hearing or visual issues. Three quarters of people in care homes have a cognitive impairment and the value of seeing a smiling face, hearing a comforting voice and reassuring touch cannot be underestimated when attempting to provide dental care. There will be some patients who may be unable to tolerate treatment safely under local anaesthesia and require sedation for urgent/ emergency dental care. For older people with comorbidities, the overall risk of sedation versus the benefit of dental care needs to be really carefully considered.'

\section{A flexible approach}

As has been pointed out, there are ways and means of adapting to the current situation before a renovation of the future. Yes, social distancing is essential, and all services will have procedures in place to abide by these government guidelines, but can community dental services really provide the flexibility necessary to make a meaningful impact on their patients?

'There are different ways social distancing can be encouraged at dental appointments', Linzi said. 'Having no-more than one patient in the waiting room at one time and encouraging staff to stay 2 metres from this patient unless when treating them. Long term, reducing the number of people attending an appointment - for example with paediatric patients allowing just one parent or carer to attend the dental appointment rather than having siblings and both parents attending - is an option that needs to be explored.'

While Vicki agreed with Linzi's suggestion, this may not be as straightforward for some patients. 'Some patients will need one or two carers to accompany them to the clinic, whereas the advice in COVID-19 urgent 
dental clinics is that patients should attend unaccompanied. This will need to be risk assessed and the carer's safety taken into consideration when carrying out preplanning for the appointment.

'The same principle applies to those patients who have dementia or severe learning disabilities. They may not be able to adhere to physical distancing or carry out infection control advice such as use of hand gel or wearing a face mask, which then puts them at higher risk for contracting COVID-19 through no fault of their own. Dental services may have to be available for longer hours over seven days due to the increase in time required for social distancing and infection control. These opening hours may not be appropriate for dependent groups who have carers, and that's before we've even discussed the additional stress it puts on the workforce.'

According to Jenny, it isn't necessarily a question of 'can'.

'I think we can, but we will need to adapt', she added. 'That may mean using buildings differently and scheduling sessions differently so that we can spread out more thinly in both place and time.

'I've been exploring remote routine dental consultations, by telephone or videocall, taking inspiration from the huge success general medical practitioners have had with video consultations since the start of the pandemic. Our team are calling it a dental 'Check-In', rather than a dental check-up or recall. The thinking is that this could substitute for a routine face-to-face consultation for many of our regularlyattending patients while lockdown remains in place. Once usual services start resuming there may yet be potential for this approach to help our vulnerable and shielded patients and their households until a vaccine has been developed.'

\section{A spike in patients?}

I have watched various outlets, including news reports and social media, shouting about the need to reopen dentistry with some intrigue. There are clearly a number of patients in pain and in need of treatment, yet either have no idea they can access care through a UTC or are aware and have been unsuccessful and sent on their merry way with antibiotics. In many cases, there are reports the latter have resorted to DIY dentistry to solve the problem.

Putting their immediate plight to one side, what it does serve to highlight is the number of patients who still seek dental care, not for routine maintenance, but when there's a problem that needs to be fixed. The BDA - amongst others in a packed field - has worked tirelessly to get prevention to the top of the agenda, not to mention stewardship on the over-prescribing of antibiotics.

In a matter of weeks, both of those appear to have returned to the bottom of the pile. Last month I asked whether NHS dentistry still had fuel in the tank and would be thrown a lifeline by an increase in post-COVID-19 patients who would no longer pay for private treatment. ${ }^{2}$ But what of the community - do they anticipate a boom in patient numbers once there is a degree of normality in the service?

\section{'There are examples of good models and care pathways of primary care and community health support for care homes residents within primary care networks that can ensure all residents have access to timely clinical care and care homes are supported'}

'I think that's very difficult to predict', Jenny added. 'We might find instead of a boom in patient numbers that parents are reluctant to bring their children for healthcare appointments because of any number of reasons: anxiety about catching the virus, not wanting their children to miss yet more school, or because they themselves need to return to work.

'But equally we could have a scramble for the first dental appointments. For my patients I want to make sure that early on we make time to see those whose need is greatest yet may not shout loudest.'

When routine treatment can resume, Linzi believes the latter of these scenarios is most likely.

'I would expect more patients would want to have treatment. So, it may be busier. However, it is very difficult to know as it is a very changing uncertain time. As Jenny has mentioned, much will be dependent on the population's attitude to COVID-19 and if they feel comfortable in their own risk/ reward decisions.'

While Mili suspects it may be slow and steady for all patients with additional needs, not only for older people, the nature of the virus may be the ultimate deterrent.

'Many older people will be anxious about leaving their homes and contracting COVID-19 until it is completely eradicated or a vaccine available', she said. 'During their isolation period older people will have become frailer and those who have been shielding will have had very low levels of exercise that will impact on their physical and mental health. Access to therapies and medical care will have been reduced therefore this culmination of issues will have an overall effect.'

Vicki added: 'It is inevitable that the need for dental treatment will have increased during the lockdown period. Dental teams will need to focus on treating urgent conditions, stabilising disease and prevention, especially if there is no routine access to AGP in dentistry for a while as the UK begins to de-escalate and rebuild dental care. Reduction of $\mathrm{R}$ value in the population, quick and accessible testing and the roll out of Test Track and Trace programme, and when available antibody testing will help to expand the ability of dental services for shielding groups.'

\section{Time for a review}

Vicki's observations about the $\mathrm{R}$ value, testing and Test Track and Trace all have long term implications for the shape of the service. With National Smile Month moving to a digital campaign in light of the current circumstances, ${ }^{3}$ is this the time for outreach programmes to be reviewed?

'Indeed they should be reviewed', Linzi said. 'For Happy Teeth Outreach, all of our school visits and study days have been postponed. Even though we cannot provide the services we usually would provide, we have worked hard to still provide a service to our target audience during the COVID-19 crisis.

'What we need is a timetable. It is unknown when we will be able to carry out our mission, and we as an organisation take our patients and volunteers safety very seriously. All outreach dental schools' projects will be completely stopped until further notice, which could adversely affect the knowledge dental students accrue at a valuable point in their development.

'We have been looking at ways we can still support children and young people with disabilities or children affected by the refugee crisis in our community. Happy Teeth Outreach has committed to still helping these children by providing oral health packs - just last week we were privileged to provide oral 
health packs for children in Bradford affected by the refugee crisis and human trafficking. These also included educational learning packs which were distributed by Bevan HealthCare centre in Bradford.

'It's only right that COVID-19 has restricted dental outreach programmes including ours - but it has fostered a new teaching style through using online teachings and a new level of connection with the teachers in the special schools. Additionally, it has enabled us to still provide oral health packs to those most in need children, and that's rewarding in any form.'

While Jenny agreed that partnership working with education and local authorities is an important way of reaching children with oral health messages and, in some places, with toothbrushing clubs and fluoride varnish programmes, she sounded a warning regarding their ongoing risk.

'These programmes must not be allowed to become a vehicle for spreading the virus' she said. 'It would be understandable if headteachers were initially cautious so we will need to be sensitive to the challenges that schools face and open to taking advice from public health colleagues. In the meantime, there are opportunities to deliver engaging preventive advice remotely via smartphones, such as using BSPD's new Dr Ranj toothbrushing videos or apps such as BrushDJ. We must use the digital options available to us.'

With the vulnerability in care homes, projects such as Mouth Care Matters need to evolve to meet the challenges posed by the current environment.

'This is the time to think about how we deliver outreach programmes in a different way, as always utilising dental care professionals including nurses, hygienists and therapists to ensure oral health is not a forgotten element of health for older people', Mili stressed. 'Now is not the time to increase the footfall in care homes for oral health training when the priority is keeping residents safe. There are no reasons why dental services cannot support care homes virtually or by phone providing advice and signposting to training resources. Carers for people in their own homes or residential care should be trained to independently support mouth care without the need for intensive dental interventions by dental teams.'

Vicki added that there are examples of good models and care pathways of primary care and community health support for care home residents within primary care networks that can ensure all residents have access to timely clinical care and care homes are supported.

'In England the aim is for all vulnerable residents to have a weekly multi team review that includes pharmacy, geriatricians, doctors and there has been mention of dentists. Dental leads should take this opportunity to get oral health included in residents' weekly health check.

'In Wales and Scotland through their national programmes - Gwen am Byth and Caring for Smiles respectively - residents in care homes have regular oral assessments and care planning by care home senior staff that highlight the need to involve local dental teams should this be required. In most areas in Wales, dental therapists have taken on the responsibility of providing dental care for residents that focuses on Delivering Better Oral Health prevention.

\section{'It is difficult to see a path where outreach programmes in the community survives inevitable cutbacks in funding. And while there is no sign they will happen, it is perhaps foolish to think otherwise. And it is those cutbacks that could have the most impact.'}

'The utilisation of skill mix for the provision of oral prevention and stabilisation of dentitions, denture provision (clinical technicians) and signposting to for more complex dental care for older people is the way forward. Given the concerns about standards of mouth care and dental access in care homes in England, now is the time to ask whether dental practices could partner with local care homes to champion oral health as they are in other nations in UK. It could potentially set the foundations for any programme post-restrictions.'

\section{Cutbacks}

It is difficult to see a path where outreach programmes in the community survive inevitable cutbacks in funding. And while there is no sign they will happen, it is perhaps foolish to think otherwise. And it is those cutbacks that could have the most impact.

'My worry as an oral health outreach provider would be the children we are not aware of', Linzi added. 'During our sessions we occasionally identify children that are not registered with a dentist and have dental decay.

'My concern is these hard to reach children may be experiencing dental pain or abscesses, but their dentist or school being unaware of their dental problems. Once schools go back this will hopefully provide a connection to those children and families who need the support, and that's what we will be doing at Happy Teeth Outreach.'

With programmes and dental teams ceasing to provide the much needed work to prevent poor oral health, Vicki believes it is inevitable that at risk groups of older people will experience dental pain and infection and be unable to receive treatment in a timely manner.

'As Mili has suggested, this was already of concern prior to COVID-19 and will be a greater issue in the recovery period where little treatment will have been provided. The consequences of poor oral health on older people can be catastrophic, they may struggle to be hydrated and maintain a healthy weight, not be able to articulate that they have oral problems and poor oral health can lead to community acquired bacterial respiratory infections. Consideration has to be given to this group. Not only are they are high-risk of contracting COVID-19, but their shortterm and long-term oral health needs will be severely hampered when it is imperative they receive quality, tailored care.'

It was left to Jenny to have the final word.

'You have pinpointed my main concern', Jenny explained. 'Our caseload includes lots of vulnerable children who previously attended only in extremis, where we've worked hard to build up trust and rapport so that they now come regularly for preventive care, and consequently have much lower treatment needs at recall than we would otherwise have expected. It would be very sad if we were to lose all that progress so we must plan to ensure that cannot happen.'

\section{References}

1. British Society of Paediatric Dentistry. COVID-19 Dental Resources for Children. Available online at: https://www.bspd.co.uk/Resources/ COVID-19/ COVID-19-Dental-Resources-for-Children (Accessed May 2020).

2. Westgarth $D$. How much longer does NHS dentistry have left? BDJ In Pract 2020; 33: 12-15.

3. Carter N. Increasing your digital influence. $B D J$ In Pract 2020; 33: 16

https://doi.org/10.1038/s41404-020-0426-4 